\title{
53
}

\section{An intelligent planning network for manufacturing systems management}

\author{
G. L. Kovács, I. Mezgár \\ Computer and Automation Research Institute, $H A S$ \\ H-1111 Budapest, Kende u. 13-17., \\ Hungary \\ Fax: 36-1-1667-503 \\ e-mail:h673kov@ella.hu
}

\begin{abstract}
The paper describes a planning network (PN) which is to assist the better harmonization and higher quality and production volume of Small and Medium Size Enterprises (SME), if they are joining their efforts by being active members of the PN. The PN means a real-time, on-line network with three basic functions. The local planning module (LPM), the co-ordination unit (CU) and the evaluation module (EM) are the three software units that cover the functions. This PN is a kind of virtual, or extended enterprise, however it is different because of the longterm co-operation of the partners, instead of working together only for 1-2 given orders. The intelligence of the PN is supported by expert systems in the CU and EM.
\end{abstract}

\section{Keywords}

Computer networks, enterprise modelling, expert systems, SME.

\section{INTRODUCTION}

In order to fulfil the market demands the flexible, effective manufacturing system architectures become more and more popular around the world. Manufacturing enterprises have a geographically distributed nature, so computer networks for production management are important feature of their operation.

There are different approaches, different names that basically cover the same idea: a flexible network of co-operating autonomous manufacturing units. The holonic manufacturing systems is an approach for a theoretical framework for 
autonomous and decentralized manufacturing organizations e.g., agile manufacturing enterprise (AME). There are different projects and developments on AME (AIMS/Stanford \& EIT, A-PRIMED/SNL, FFC/MIT, etc.) that use different names for the AME, e.g., "extended enterprise" or "virtual enterprise (VE)".

The structure of a virtual enterprise can be seen as a heirarchy, in that it is a temporary, goal-oriented aggregation of several individual enterprises. Each virtual enterprise is created to pursue a specific business objective, and remains in life for as long as this objective can be pursued. After that, the individual nodes resume their independence from each other. The resource of a node that was previously allocated to the expired business is re-directed toward the node's individual goals, or toward other virtual enterprises it may have joined.

The virtual enterprise model has been developed for decomposing large companies that have enough financial, human and organizational resources. The decomposition is based on functions that can result in replications across the network.

Many geographic regions can be characterised with special industrial organisational structures called Small and Medium Sized Enterprise (SME). These SMEs are well-known for their dynamic behaviour, but their prosperity, and sometimes their existence, is continuously exposed to risks of the limited investment capability, the difficulty of diverting skilled personnel from day-by-day activities, to undertake process re-engineering initiatives, the lack of advanced planning support tools specifically conceived for them. Because of these reasons it is even a harder problem for the SMEs to give proper answer for the market challenges. The co-ordination of the different SME's production in case of producing a product jointly generates similar problems as in the case of large firms, but the solution is different (Bonfatti 3, 1996).

In a distributed manufacturing environment the production planning has a key role. The co-ordination of the orders, the optimal assignment of the different resources in a co-operative production of several SMEs is a very difficult task. In an SME network there is a strong need of a network model that is based on the planning strategy (Bonfatti 1, 1994). Another important goal during the software development is the product independence, i.e. the network model has to be general enough, to be able to apply it in a wide range of different products, in different production environments.

Our SME network development work is done in the frame of the "PLENT"ESPRIT Project No. 20723 with the participation of firms from Italy, Greece, Hungary and Spain. The main goal of the project is to support organizations that manufacture mechanical parts and products in SME-like production environments. The paper shortly describes a special application of the manufacturing network model for agriculture production (oil and seed production) to demonstrate the applicability of this management philosophy and network model in fully different fields. 


\section{THE PLENT PROJECT}

\subsection{Comparison of the holonic/virtual and the PLENT approach}

In the PLENT project a practical approach of the virtual enterprise has been realized for SMEs. In order to give a better understanding the PLENT approach will be shortly introduced in comparison with the classical holonic theory as it was discussed by Koestler (1989).

The holonic manufacturing system is a system of co-operating holons that are organized to achieve a production goal. This system integrates the whole range of manufacturing activities from order booking through design, production and marketing to form an autonomous and decentralized manufacturing organization.

According to the holonic organization paradigm the manufacturing environment is going to transform itself into a holonic system as the openness, the flexibility and the similarity in building blocks are vital advantages. The virtual enterprise is a practical instantiation of the holonic paradigm in the form of a multilayer, open, flexible (through continuous, dynamic re-configuration) network.

As the PLENT project aims to coordinate the production of SMEs the holonic approach had to be modified. The SMEs have limited financial and human resources. Usually they have no advanced planning support tools that are essential for a fully open VE. There is also a historical background namely the traditional individualistic attitude of SMEs. This means the facing of marketing, design, engineering, technological innovation and purchasing problems alone. Handling the SME network as a limited number of independent nodes has the following advantages:

- More complex and technologically advanced products can be realised by putting together skills and design capabilities of the network nodes.

- Higher manufacturing volumes are obtained by cumulating node capacities, especially if two or more nodes concur in performing the same manufacturing phases.

- Fluctuations of market demand volumes are better borne by sharing workload peaks and shortages among the network nodes.

- The limited resources of each SME are better spent in technological innovation and process re-engineering rather than in hard competition with other SMEs.

In consideration of the requirements expressed by SMEs, it is possible to compare the PLENT idea of virtual enterprise with that currently derived from the holonic theory (Bonfatti 2, 1995). Indeed, a number of properties commonly attributed to the virtual enterprise are no longer valid when transferred to the PLENT organisation model. The most significant differences between the two approaches are listed: 
- Functional specialization versus flexibility;

- System duplication versus identity preservation;

- Temporary versus stable aggregation;

- Local versus global network co-ordination.

In conclusion, the PLENT VE model project focuses on distributed production management in a stable network of co-operating SMEs. The initial phases of network design and creation, as well as the pre-existing relationships that made cooperation possible, are outside the project scope. A detailed description can be found in Deliverable D2 (1996).

\subsection{PLENT manufacturing network model}

In order to achieve the required network timeliness and co-ordination neutrality, the PLENT network organisation must be supported by expressly designed planning tools. In particular, three software modules are required: a co-ordination module, a local planning module and a performance evaluation module.

- Co-ordination module

The co-ordination module realises the network planning policy. The coordination module is responsible for controlling the connection outside the network, for keeping the contact with the customers, for selecting the proper nodes (production units) and for distributing production tasks to them.

- Local planning module

The local planning module supports the network-related activities in charge of each node, aimed at managing and communicating delivery plans and delivery conditions with the co-ordination unit and the supplier and client nodes. The local planning modules realise the network controlling tasks of the node, in order to fulfil the goals of the management and communication, and deliver the status information of the node for the other nodes.

- Performance evaluation module

The performance evaluation module is used by the co-ordinating unit to calculate node reliability starting from measures of their past behaviours, and network reaction capability by simulating possible scenarios. The performance evaluation module supports the work of the co-ordination unit in selecting the best node for fulfilling a task. This node generates different measures, statistics; estimations based on the past behaviour of the nodes.

Based on the PLENT VE approach the four different national networks of the project participants are under development. The product profiles of the national networks are different, demonstrating by this fact that this type of virtual manufacturing approach can be applied under different circumstances. In the following chapter the main characteristics and the organization of the Hungarian Network will be presented. 


\section{DESCRIPTION OF THE HUNGARIAN NETWORK}

The main activities in the Hungarian Network (HN) are connected to the coordination unit that is an investment Co. These are: financing oil seed growing on plantations, seed-oil production and trading with the products which are the seeds and the seed-oils. The products as physical objects are seeds, seed-oils and contracts for production with farms and factories, and contracts for trading with trading houses. The market of the products is in Hungary and abroad. The main functionality is contracting partners to produce and to sell and buy. There is a management and a staff to prepare contracts using advanced forecasting and trend analysis tools. The product delivery cycle is unique: no cycle for the contract, however there is a special cycle for the seeds and oils covered by the contracts, as financing starts very early, 3-12 months before harvesting. There are three different profiles of individual nodes in the network:

- Big farms and oil pressing factories. Their activities include market research, approval, contract preparation, contracting, growing the seeds on the plantations and delivery of seeds or pressed oils.

- Small farms and seed-producers. Their activities are basically the same as above, but they are organized in one big group.

- Trading houses. Their activities include the seed and oil purchasing, the selling of the purchased goods and the cashing of the money.

For detailed description and analysis of the HN please see in Deliverable D01 (1996). The evaluation module is an independent software node in the first pilot implementation.

\section{PLANNING REQUIREMENTS FOR THE HN}

The PN means a real-time, on-line network with three basic functions. The intelligence of the Hungarian PN is supported by expert systems in the CU and EM. The CU and the EM software may be included into any of the nodes, or they may be independently implemented, a LPM must belong to all participating nodes. The tasks of the expert systems are double; they solve the planning problems of the network and parallel handle/control the complex communication among the $\mathrm{CU}$ and the EMs.

Before starting the ES development the planning requirements for the ESs have to be investigated very carefully, in order to avoid critical problems later.

\subsection{Planning requirements for the co-ordination unit}

- Subcontracted seed and oil production orders monitoring. It is interesting for the co-ordination unit be able to consult in any moment the production order's list launched for a given order. In addition it shows all 
the associated data with each order, like the following: start date, forecast delivery, progress state of the work, etc.

- Node workload monitoring

The co-ordination unit could see in any moment the forecast workload for each node for a period of time. This information is very important for the workload distribution between the nodes. In order to get this, it is necessary to update periodically the information used by the co-ordination unit.

- Monitoring of the accepted and pending subcontracting offers

The co-ordination unit requires having a list of the subcontracting offers for each special product and partner. So the co-ordination unit could control the state of each offer like accepted, pending of acceptance, to be sent to the node.

- Sending of the planning of the subcontracted groups to the nodes for their fulfilment.

The node needs to receive the current information and the group planning. The co-ordination unit defines some milestones. The node would establish the milestone's date and this information would return to the co-ordination unit.

- Linking between the co-ordination unit planning and the node planning.

The co-ordination unit divides the planning in some activities. These activities might be used in the node planning, so they could be controlled from the co-ordination unit that should know the manufacturing progress. This information exchange might be in an electronic environment or similar. So each time the co-ordination unit connects with a node it is getting the last information.

\section{Task distribution and task assignment criteria}

As it was mentioned above, in principle all nodes are capable of accomplishing any of the production tasks. Due to historical reasons and work sharing it is normal to have a distribution as not all partners have oil pressing facilities among producers, and the trading houses are different in relations and in their main lines (special seeds, or oils, or both).

\section{Workload distribution}

The workload distribution is accomplished when the needs calculations are performed. This is the moment when the seed growing, oil production and marketing launches for each group are assigned. The local planning task is made using different methods (not necessarily computerized) at different sites.

\subsection{Planning requirements for the nodes}

- Forecasting, trends and market analysis.

The co-ordination unit and the node's computer systems are similar, so it is interesting to have a more automatic information exchange method.

The first application for this automation might be the exchange of the forecasting and analysis data from the co-ordination unit to the nodes. With 
this automation some actual steps could be avoided and some benefits, like: paper reduction, reduce the time required in the information entry, and less writing errors, etc. might be obtained like.

- Access to the co-ordination general planning in order to foresee the workload. After making the first planning from the co-ordination unit this information might be sent to each node telling them the potential production/trading needs and the estimated dates. With this information the node might foresee their necessary production/trading for a period of time. One benefit of this policy would be that the nodes would avoid or would reduce some bottlenecks in their production.

- Anticipated prevision of the potential orders and workload. This point would be a consequence of the previous point.

- Group design evolution in the co-ordination unit.

If the node knows in advance his workload, the node might take some information from the co-ordination unit about the state of the design activities related with the product group it has to produce. So depending upon the design state the node might foresee potential delays in the preparation and replanning its local planning.

- To dispose a local planning tool with a finite capacity. It might be interesting in order to optimize the workload.

- To define the verification cards between the co-ordination unit and the nodes. The co-ordination unit sends to the nodes all the required information about the production group. Besides the co-ordination unit sends to the nodes a verification card, where it defines the verification requirements. So the node might participate in the verification card definition in order to optimize the quality process.

In Table 1, a few rules/actions are introduced for re-planning the original production plan in case of special, unscheduled events (problems). 
Table 1. Some selected rules/actions for re-planning the original production plan

\begin{tabular}{|c|c|}
\hline Problem & Action \\
\hline $\begin{array}{l}\text { The node refuses the appraised } \\
\text { offer. }\end{array}$ & $\begin{array}{l}\text { 1. Subcontract to another node. } \\
\text { 2. Make new offer appraises. } \\
\text { 3. Production subcontracting to other } \\
\text { nodes, out of the network. }\end{array}$ \\
\hline $\begin{array}{l}\text { The node can not finish the work } \\
\text { on time. }\end{array}$ & $\begin{array}{l}\text { 1. To urge the node in order to finish } \\
\text { the work on time. } \\
\text { 2. Depending of the final evolution. } \\
\text { 2.1. If it not suppose perturbation in } \\
\text { the final business, the activity is } \\
\text { re-planned. } \\
\text { 2.2. If it suppose perturbation in the } \\
\text { final business, the activity is re- } \\
\text { planned and the customer is } \\
\text { advised and explained the reasons } \\
\text { and the future actions. }\end{array}$ \\
\hline $\begin{array}{l}\text { The node's supplier is out of the } \\
\text { delivery time. }\end{array}$ & $\begin{array}{l}\text { 1. Ask to the customer to urge the } \\
\text { supplier in order to supply on time. } \\
\text { 2. Ask to the co-ordination unit to } \\
\text { urge the supplier in order to supply } \\
\text { on time. }\end{array}$ \\
\hline Customer modification. & $\begin{array}{l}\text { 1. Re-negotiate with the customer. } \\
\text { 2. To communicate to the nodes. } \\
\text { 3. Project re-planning. }\end{array}$ \\
\hline $\begin{array}{l}\text { Disfunction on the production } \\
\text { groups. }\end{array}$ & $\begin{array}{l}\text { 1. If it is very expensive, advice to the } \\
\text { node in order to re-organize it in } \\
\text { the co-ordination unit. } \\
\text { 2. If it is simple, it is re-organized by } \\
\text { the co-ordination unit. }\end{array}$ \\
\hline
\end{tabular}




\subsection{Communication and information exchange}

In case of a planning network the definition of the regular communication relations, routes in the design phase of the network are very important. Table 2 shows part of the communication and information exchange requirements with the following assumptions (Information exchange is really bi-directional in most cases, this is shown by multiple arrows):

- There is no communication between production and trade nodes. They communicate only with the co-ordination unit

- The customer has no direct contact to the production and trade nodes, only to the co-ordination unit

- The co-ordination unit is the investment Co. in our special case (as soon as an independent co-ordination unit will be established it would be changed to a more special local node)

Table 2. A part of the information exchange among the nodes and the $\mathrm{CU}$

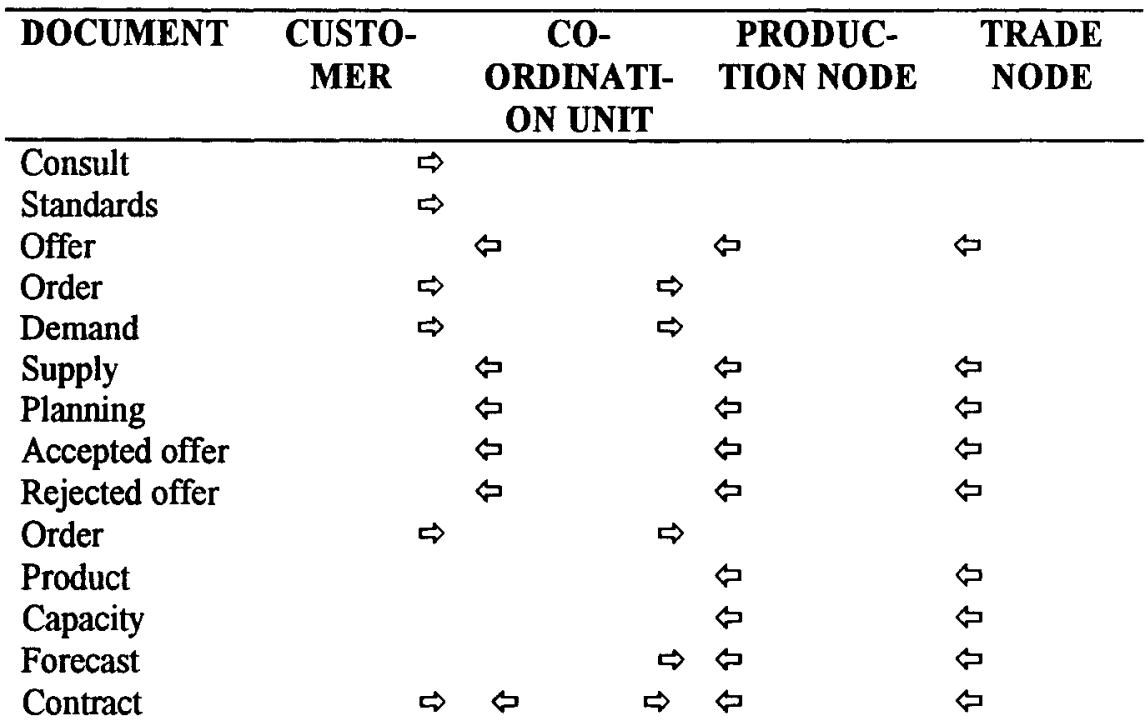

\subsection{Expected benefits}

The main advantage of using the described network is the more effective usage of each SMEs (farms, oil pressing factories) resources, faster reaction for market demands while keeping the individual characteristic of each node (farm). Through this coordination the production of a very unstable, unreliable (working with high risk) production field, the agricultural production can become more reliable, more safe both for the customer and the farmers that are members of the network. The reliability leads to more orders, stabilized and higher income, less costs i.e. more profit. The expected costs of the investment (network installation and software) are 
low, to learn the applied information technology needs only short time, so the PLENT manufacturing network has good chances for success in agricultural field as well.

\section{CONCLUSIONS}

In the PLENT approach, a production network is conceived as a set of operational nodes with equal rights, co-ordinated by a central unit that interacts with customers, distributes tasks to nodes on the basis of prefixed criteria and up-to-date information on node state and reliability, and informs each node on its position in the network with respect to the manufacturing process. These tasks are realized by an expert system, that deals both with the planning/re-planning and the control of communication among $\mathrm{CU}$ and the nodes.

This new approach for SME network coordination is very promisable, because it offers the advantages of the holonic manufacturing paradigm parallel giving the possibility of keeping the traditional individualism of SMEs. The realization of the method is going on in four countries involving SMEs from three different fields of production (in machine- and textile industry, in agriculture).

The results achieved so far prove that it is possible to develop network based co-ordination software for SMEs that is general enough to be applied in fully different production fields. The early experiments show that the ISDN-based Hungarian Network has a good perspective. The program development phase has already started using Visual $\mathrm{C}++$ and SYBASE.

\section{REFERENCES}

Bonfatti 1, F., Monari, P. D. and Paganelli, P. (1994). Modeling manufacturing resources and activities: an ontology. In: Proc. of ICCIM '95 Intern.Conf., Computer Integrated Manufacturing, Singapore, 1995, Edited by J. Winsor, etc., p.p. 101-108. World Scientific, Singapore.

Bonfatti 2, F. and Monari, P. D. (1995) Planning small-medium enterprise networks. In: Proc. of the IMPDE Internal. Conf., Edinburgh, 1995, p.p. 13-22, Sponsored by European Commission DGIII, ESPRIT Consortium 9245.

Bonfatti 3, F., Monari, P. D. and Paganelli, P. (1996) Co-ordination Functions in a SME Network. In: Proc. of BASYS '96 Intern. Conf., Balanced Automation Systems II, Lisbon, 1996, Edited by L.M. Camarinha-Matos etc., p.p. 383-390. Chapman \& Hall, London.

Koestler A. (1989) The Ghost in The Machine, Arkana Books.

PLENT ESPRIT project 20723, Deliverable D01 (1996). Requirement Specification and Analysis.

PLENT ESPRIT project 20723, Deliverable D02 (1996). Planning Policy Formalisation. 


\section{BIOGRAPHY}

George L. Kovács graduated at the Technical University of Budapest, Faculty of Electrical Engineering, 1966. Dr. degree (Ph.D.) at the same university, 1976. Candidate of sciences degree at the Hungarian Academy of Sciences, 1978 (CAD/CAM in electronics). Research fellow in the Computer and Automation Institute of the Hungarian Academy of Sciences since 1966. He got his habilitation and Prof. degree at the Technical Univ. of Budapest in 1995. Recently head of CIM Research Laboratory since 1990. Visiting researcher in the USA (one year), Soviet Union (two years), West Germany (one year) and visiting professor in Mexico (six months). In 1994 he was visiting professor in Italy, University of Trento. More than 100 scientific publications. Deputy chairman of the Eastern European MAP/TOP Interest Group, member of several scientific organizations. Scientific interest: CIM, computer aided design and manufacturing, expert and hybrid systems, industrial networks.

István Mezgár [Diploma in m.e.: 1977, special Diploma in automation engineering: 1988, dr.Univ in m.e.: 1990, all from the Technical University of Budapest (TU), C.Sc. in m.e.: 1995, from the Hungarian Academy of Sciences] is a senior researcher at the CIM Research Laboratory at the Computer and Automation Research Institute, Hungarian Academy of Sciences. Visiting researcher/professor at the University of Trento, at the University of Genoa (Italy, in 1989-90), at the University of Waikato (New Zealand, in 1995), and at the CAD/CAM Research Laboratory, Korea Institute of Science and Technology (South-Korea, in 1995-96). IPC member of several international IFAC, IFIP conferences. More than 60 scientific publications. His current interests focus on manufacturing automation, integration of product and enterprise models, agile manufacturing systems, design methodologies (e.g. concurrent engineering) and complex quality control. 